\title{
Discussion on upgrading construction of environmental protection hotline driven by Al technology
}

\author{
Bin Wang ${ }^{1}$, Jian $\mathrm{Li}^{1}$, Shengnan Sun ${ }^{1}$, Lu Wang ${ }^{1}$, Xiaoyuan Wan ${ }^{1}$, Zheying Yu ${ }^{1}$ \\ ${ }^{1}$ Beijing SinoVoice Technology Co.,Ltd,100089,BeiJing
}

\begin{abstract}
Enhancing the public's awareness of environmental protection is an important measure to achieve the goal of carbon peak and carbon neutrality. This article takes the improvement of public participation in environmental protection as the starting point, discusses the enthusiasm of the public in environmental protection and its legal channels for reporting and complaining of environmental pollution, summarizes the achievements and existing problems of the construction of complaint channels, and proposes to improve environmental protection reports. Channel countermeasures and suggestions, build a reporting channel of environmental protection with temperature through artificial intelligence technology, increase public awareness and willingness to participate, and promote the development of environmental protection.
\end{abstract}

\section{Introduction}

President Xi Jinping put forward the idea of "beautiful scenery and green hills and clear hills" in 2005. With the development of the times, this concept has shown its strong vitality. Emphasizing that "green water and green mountains are golden mountains and silver mountains" is to maintain the fine balance between economic development and ecological environment as far as possible, and take the path of ecological priority and green development ${ }^{[1]}$. Along this way from green water and green mountains, China will surely have both the prosperity of modern civilization and the beauty of ecological civilization in the future. In the fourth session of the 13th National People's Congress, Premier Li Keqiang demanded: accurately grasp the new development stage, thoroughly implement the new development concept, speed up the construction of a new development pattern, promote high-quality development, and make a good start for building a socialist modern country in an all-round way ${ }^{[2]}$. China will put the construction of ecological civilization in a more prominent position, promote green development, and promote the harmonious coexistence between man and nature. We need to systematically grasp the strategic task of the construction of ecological civilization during the "fourteenth five year plan" period.

Environment is the basis of human survival, and the destruction of environment poses a threat to the survival and development of human society. The newly revised environmental protection law of the people's Republic of China, implemented on January $1^{\text {st }} 2015$, clearly stipulates that citizens, legal persons and other organizations shall enjoy the right to obtain environmental information, participate in and supervise environmental protection according to law. This provides legal basis for public participation in environmental protection. To improve the public awareness of environmental protection participation and enhance the public participation degree is an effective way to prevent the transformation of environmental risk to social risk. However, due to the lack of participation in environmental protection, the government information is not open and transparent, ignoring the public interest demands and participation wishes, and there is no corresponding response mechanism, which makes environmental risk into social risk $^{[3]}$."The State Council's opinions on strengthening the key work of environmental protection" are clear: to solve the outstanding environmental problems that affect the scientific development and damage the health of the masses, it also puts forward the "establishment and improvement of the environmental protection reporting system", unblocking the channels of public reports, reflecting the public opinion and people's situation in time, and raising the efficiency of accepting \&processing the report.

\section{Construction achievements}

The Ministry of Ecology Environment of the People's Republic of China (formerly the Ministry of Environmental Protection of the People's Republic of China) published a hotline for environmental protection report on June $5^{\text {th }}, 2009$, and successively opened Wechat report and internet report. In 2019, the national 12369 environmental protection report network management platform (hereinafter referred to "Internet platform") received 531176 reports from the public, a year-on-year decline of $25.2 \%$, from which,270451 phone reports, 195950 Wechat reports, 62240 Internet reports and 2535 other ways, including $26.0 \%$ drop in telephone

\footnotetext{
${ }^{*}$ WangBin: wangbin@sinovoice.com.cn
} 
reports, $21.6 \%$ decrease in Wechat reports and 22.9\% decrease in internet reports. See Table 1 for details ${ }^{[4]}$.

Table1. Acceptance of national environmental protection reports in 2019.

\begin{tabular}{|c|c|c|c|}
\hline \multirow{2}{*}{ Source of report } & \multicolumn{3}{|c|}{2019} \\
\cline { 2 - 4 } & Reports & Percentage & Growth rate \\
\hline Phone & 270451 & $50.90 \%$ & $-26.00 \%$ \\
\hline Wechat & 195950 & $36.90 \%$ & $-21.60 \%$ \\
\hline Internet & 62240 & $11.70 \%$ & $-22.90 \%$ \\
\hline Other ways & 2535 & $0.50 \%$ & - \\
\hline Total & 531176 & - & $-25.20 \%$ \\
\hline
\end{tabular}

The published of the environmental reporting hotline has greatly enhanced the enthusiasm of the public to participate in environmental protection, and protected the legitimate rights and interests of the public to report and complains about environmental pollution behaviors. The acceptance of environmental reporting hotline takes up a lot of time of environmental protection staff, and because of the relatively fall behind system construction and uneven quality of staff, the acceptance efficiency and acceptance quality are affected.

\section{Existing Problems}

\subsection{The reporting hotline system is fall behind}

The environmental reporting hotline was published on June $5^{\text {th }}$,2009. After 2015, the Ministry of Ecology Environment has successively onlined Wechat reporting,internet reporting and other ways, with scattered system and relatively fall behind technology. In order to implement the opinions of the State Council on strengthening the key work of environmental protection, unblock the reporting channels of the masses,timely reflect the public opinion, and improve the efficiency of reporting acceptance,the existing system must be upgraded through AI technologies such as Automatic Speech Recognition (ASR), Natural language understanding (NLU), Text To Speech (TTS) and Big Data Analysis(BDA), etc.

\subsection{The hotline service staff is obviously insufficient}

The shortage of hotline staff is manifested in two aspects: one is the lack of full-time staff, which is difficult to ensure a 24-hour timely response; the other is that the hotline service staff do not have professional quality, only act as the role of the operator, and it is difficult to give a correct reply to the public in time when the public asks professional questions. In the process of acceptance of environmental protection report, it is inevitable that there are some problems, such as inaccurate service terms, random information reply and irregular feedback, which will have a negative impact on the government's credibility and administrative efficiency ${ }^{[5]}$.

\section{Countermeasures and suggestions}

With the full help of artificial intelligence, big data, $5 \mathrm{~g}$ and other frontier technologies, the way of public participation in environmental protection is improved. Through Automatic Speech Recognition (ASR) , Natural Language Understanding (NLU), Text To Speech (TTS) and Big Data Analysis(BDA) and other artificial intelligence technologies, the upgrading construction of environmental protection reporting hotline is driven. Combined with the business attributes of environmental protection reporting hotline, the workflow of the system is designed to realize the intelligent accepting and processing, intelligent supervision, intelligent management of reporting, intelligence feedback, report intelligence analysis, and earnestly achieve 4 points " 1 st accepting and processing every reoprt $; 2^{\text {nd }}$ any illegal will be investigated $; 3^{\text {rd }}$ all investigation will have results, $4^{\text {th }}$ resultswill be feedback to public".

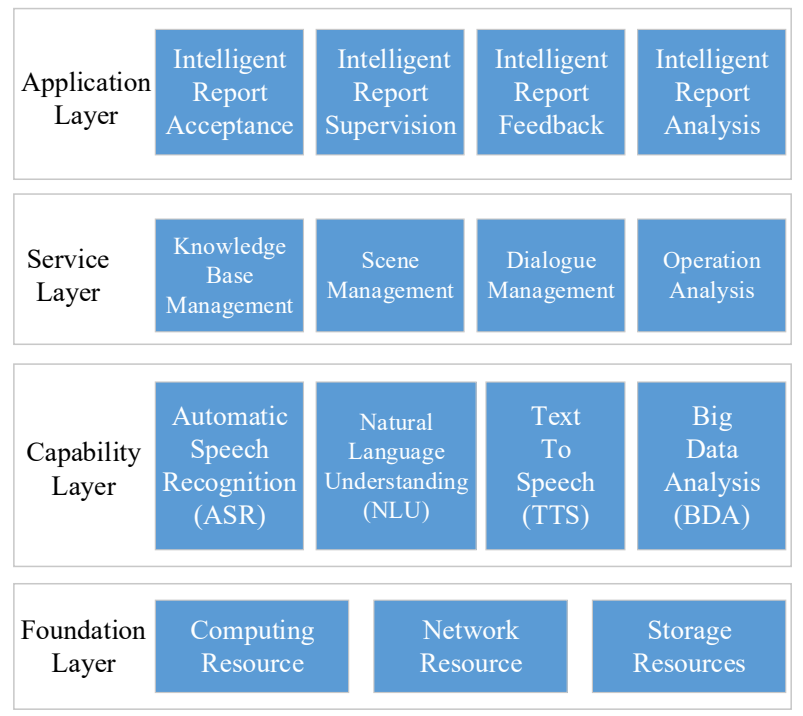

Figure 1. System architecture of intelligent environmental protection hotline

Basic Layer: Provide computing resources, network resources and storage resources for intelligent environmental reporting hotline.

Capability Layer: provide intelligent voice recognition, intelligent semantic understanding, intelligent speech synthesis, big data analysis and other AI core technical capabilities for intelligent environmental protection reporting hotline.

Service Layer: Provide environmental knowledge base management, environmental business scenario management, hotline dialogue management and operation 
analysis services to meet the needs of intelligent environmental hotline.

Application Layer: Build artificial intelligence application scenarios such as intelligent report acceptance, intelligent report supervision, intelligent report feedback and intelligent report analysis, and use artificial intelligence technology to improve the service efficiency and quality of environmental protection report hotline.

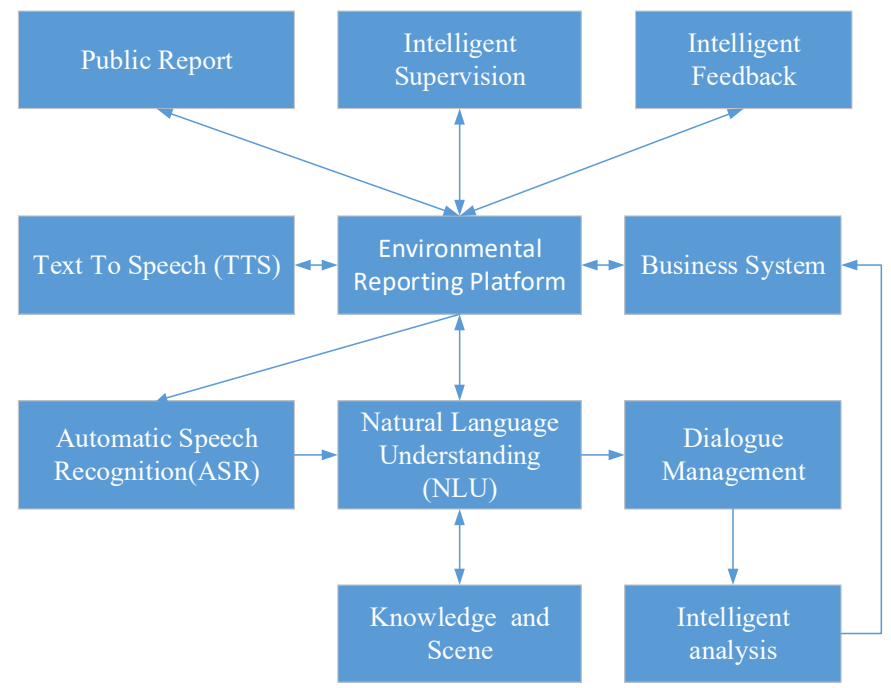

Figure 2. Work flow chart of intelligent environmental protection reporting hotline

\subsection{Intelligent Report Accepting and Processing}

The intelligent report accepting and processing system included AI technologies such as Automatic Speech recognition (ASR) , Natural language understanding (NLU) and Text To Speech (TTS) as the core has been mature. The accuracy of Q \& A is higher than $90 \%$, and the average response speed is less than $200 \mathrm{~ms}$. The system can integrate multiple channels such as telephone, website, Wechat, app and H5 to unify and expand the public report channels. By upgrading the existing environmental protection reporting hotline, we can build a AI platform for environmental protection reporting hotline, intelligent interaction, automatically enter work orders, and assist or replace manual work to accepting and processing public environmental protection report. The system can keep online for $7 * 24$ hours, improve the efficiency of staff work, greatly reduce the cost of customer service personnel, and improve the timeliness and professionalism of problem response, Improve service efficiency and customer satisfaction.

\subsection{Intelligent Report Supervision}

Through the unified design of the system architecture, the problem of system data silos is completely solved, and the integration of reporting data and business processing information are realized. The intelligent voice outbound system is used to automatically judge the case level and waiting time, and to automatically supervise and remind the security inspection of overtime reports, so as to timely follow up the progress of the case, focus on ensuring the timeliness of the acceptance and completion of environmental protection reports, and improve the quality of report information feedback.

\subsection{Intelligent Report Feedback}

After the case is handled, it can feed back the processing results to the informants in batches through the intelligent voice outbound system, and conduct the satisfaction survey of the processing results at the same time. If the service results are satisfied, it will be automatically completed and filed. If the service results are not satisfied, it will be transferred to the relevant departments again. In addition, the Enviromental Reporting Platform can also complete the public's problem consultation work by itself, and can also realize self-service transfer to manual customer service, so as to improve public satisfaction and government credibility.

\subsection{Intelligent Report Analysis}

Through the core technologies of Automatic Speech Recognition (ASR) ,Natural Language Understanding (NLU), Text To Speech (TTS) and Big Data Analysis(BDA), the public report information is analyzed. The system realizes the full transcribing of hotline recordings of all levels of units, excavates and analyzes the voice content, timely discovers service abnormalities, missing reports, concealment and other situations, and conducts data mining and analysis through reporting hot spot analysis, repeated call analysis, user portrait analysis, public opinion analysis and other dimensions, so as to deeply explore the public reporting demands, so as to optimize service quality, improve management efficiency, improve the quality of service, improve the quality of service, and improve the quality of service last but not least to provide important data for policy-making. 


\section{Summary}

Based on AI core technologies such as Automatic Speech Recognition (ASR) ,Natural Language Understanding (NLU), Text To Speech (TTS) and Big Data Analysis(BDA), through unified architecture design, intellectualization are used to assist or replace the hotline service personnel. At the same time, the problem of data silos between various systems is solved, the automatic flow of business data is realized, and the potential value of data is mined. Through the intelligent upgrading construction of reporting hotline, build convenient environmental reporting hotline, and effectively solve the problems such as poor accepting and processing of reports, limited acceptance capacity of staff, inaccurate service terms, random information reply, and nonstandard feedback. Enhance the awareness and willingness of public participation and promote the development of environmental protection.

\section{References}

1. Ministry of ecological environment of the people's Republic of China.Party School of the Central Committee of C.P.C(National Academy of Governance).[ EB/OL].(2020-08-11). https://www. ccps .gov.cn/1lwx/202008/t20200811_142737.shtml.

2. Shanghai Oriental newspaper Co., Ltd. Interpretation of the government work report, ecological civilization construction and carbon emission reduction goals: do a good job in integration, grasp the direction of coordination. [ EB/OL]. (2021-0308). https://m.thepaper.cn/bai- jiahao_11619805

3. B.H.Li, D.M.Zhu. Research on the influencing factors of public participation in environmental protection[J]. Ournal of Shanghai University (SOCIAL SCIENCE EDITION) 1(2020):118-128.

4. Ministry of Ecology and Environment of the People's Republic of China. The Ministry of Ecology and Environment announced the national "12369" environmental protection report in 2019. [ EB/OL]. (2020-05-13). http://www.mee.gov.cn/g$\mathrm{kml} / \mathrm{hbb} / \mathrm{qt} / 201612 / \mathrm{t} 20161222$ 369409.htm.

5. S.F.Niu, X.L.Wei. Give full play to the role of the main Way of the hotline, and earnestly safeguard the people's environmental rights and interests. [J]. Regional Economy and Industrial Economy,2013:1314. 